\title{
乌s \\ Energy slicing analysis for time-resolved measurement of electron-beam properties
}

\author{
E. Allaria, G. De Ninno, S. Di Mitri, W. M. Fawley, E. Ferrari, L. Fröhlich, G. Penco, \\ P. Sigalotti, S. Spampinati, C. Spezzani, and M. Trovò \\ Elettra-Sincrotrone Trieste, Strada Statale 14, 34149 Basovizza, Trieste, Italy
}

(Received 24 June 2013; published 31 January 2014)

\begin{abstract}
We present a new diagnostic method allowing one to reconstruct certain properties of a relativistic electron beam and of a laser pulse by looking at the result of their interaction. The latter takes place within an undulator and results in energy modulation of electrons. By taking advantage of the correlation between time and electron-beam energy, measurements of the electron-beam length, current and energy spread are possible simultaneously. Moreover, the scheme allows an accurate measurement of the timing jitter between the electron beam and the external laser and also of the laser's actual pulse length at the interaction point. Experimental demonstration of the last two measurements is reported in this paper. Finally, this diagnostic method provides an independent measure of the energy transferred from the electron beam to free-electron laser light produced when seeded electrons are injected into a long undulator. Experimental results obtained at the FERMI free-electron laser are presented after a description of the proposed technique.
\end{abstract}

DOI: 10.1103/PhysRevSTAB.17.010704

PACS numbers: 41.60.Cr, 41.75.Ht

Single-pass free-electron lasers (FELs) are powerful tools for investigating the fundamental properties of matter, giving unprecedented spectral and temporal resolution [1]. Efficient FEL operation, as in the existing facilities [2-6], relies on the generation of high brightness electron beam, with very low normalized transverse emittance $(<1 \mathrm{~mm} \mathrm{mrad})$ and high peak current $(\sim \mathrm{kA})$.

The characterization and optimization of such high brightness beams require the development and implementation of state-of-the-art diagnostics, such as scintillating screens [7], wire scanners [8], and optical transition radiation $[9,10]$ monitors for measuring the transverse charge distribution.

Concerning longitudinal diagnostics, coherent radiation emitted from the electron bunches is routinely used for relative measurements of the bunch length [11], although some absolute evaluations have been demonstrated to be possible [12]. Measurements can be based on pyroelectric detectors, diodes, or similar devices [13], or by means of more complex setups that allow obtaining also spectral information [14,15]. For accurate reconstruction of the electron-beam current profile, the optical replica synthesizer was proposed in [16]. Alternatively, transversely deflecting cavities [17] are powerful but expensive tools that allow the reconstruction of the current profile. Finally, electro-optical sampling techniques [18] strive to provide a

*enrico.allaria@elettra.eu

Published by the American Physical Society under the terms of the Creative Commons Attribution 3.0 License. Further distribution of this work must maintain attribution to the author(s) and the published articles title, journal citation, and DOI. nondestructive measurement of the longitudinal charge distribution within the bunch.

Electron bunch arrival time jitter is another critical parameter for the operation of FELs. For FELs based on self-amplified spontaneous emission, such jitter is directly converted into a temporal jitter of the emitted FEL radiation pulse. Electron timing jitter is determined by the stability of several accelerator components (e.g., linac rf phase and amplitude, injector laser time jitter, etc.). It usually ranges from a few tens to several hundreds of femtoseconds [19-22]. Often being larger than the FEL pulse duration, these values can seriously limit the possibility of certain pump-probe experiments. Therefore, special diagnostics have been proposed [23] and implemented [21,24] to monitor simultaneously the electron beam and the FEL pulse jitter, in order to allow correction of the collected pump-probe data.

Timing jitter can be mitigated by the use of an external seed to initiate the FEL process [25]. In seeded FELs that generally use a seed laser pulse shorter than the electron beam, the timing of the FEL is linked to that of the seed laser and not to the electron beam. This allows a significant improvement of the time jitter between the FEL pulse and an external laser in pump-probe configurations. However, also for a seeded configuration, the electron-beam timing jitter and, more importantly, the relative timing jitter between the electron beam and the seed laser are important because they set the minimum required duration of the homogenous portion of the electron bunch (i.e., that with constant emittance, flat current, and energy distributions). If the latter is small compared to jitter, the interaction will often occur in a low-quality region and the output FEL 
pulse energy will be strongly reduced or even suppressed affecting the FEL power stability. Jitter measurements are even more important for multiple stage FELs, where different portions of the electron bunch are used for each stage [26-28].

In this paper a new method is proposed to simultaneously measure the time-varying parameters of the electron beam and the arrival time jitter between the electron beam and the external laser.

While we primarily focus on timing jitter measurements, the proposed scheme is also capable of providing additional detailed information on several laser and electron-beam properties, as well as on the FEL process. Based on the laser-induced local modifications to the electron-beam phase space that are easily detectable in an energy spectrometer, the scheme is suitable for time-resolved characterization of the beams. Since the analysis of the electron-beam energy spectra can be done at the beam dump downstream of the FEL undulators, all aforementioned measurements can be carried out in a noninvasive way and offer new possibilities to study the FEL effects on the electron-beam dynamics. Although the present work was developed in the framework of an externally seeded FEL, the same technique can be implemented in other (not necessarily externally seeded) FEL facilities, once the electron-external laser interaction is ensured. This applies, e.g., to the FLASH facility where there is an existing optical replica diagnostic $[3,20]$.

The reported experiment was carried out at FERMI $[4,29]$. As shown in Fig. 1, FERMI comprises a photoinjector, the main linac that accelerates the beam up to $1.2 \mathrm{GeV}$, a magnetic chicane for compressing the beam from $10 \mathrm{ps}$ duration down to less than $1 \mathrm{ps}$, a transport line to the undulators, and finally the undulator chain, where the FEL radiation is generated. Downstream of the undulators, the FEL radiation is transported to the user experimental area, while the electron beam is sent to a dump along an energy-dispersive transport line.

After having been generated in the photoinjector [30], the electron bunch is accelerated to approximately $300 \mathrm{MeV}$ energy by a linac operated off crest in order to induce a monotonic and approximately linear energy variation from the head to the tail of the bunch. This correlated energy spread is required for bunch compression in the magnetic chicane (BC1). Following this chicane, the second part of the linac is run on crest to reach the maximum final energy. The linac is followed by an achromatic transfer line to the undulator complex where the electron beam is seeded by an external laser. The electron beam is finally driven into the main beam dump (MBD) spectrometer line. Another spectrometer line, called the diagnostic beam dump (DBD), is used at the linac end (before the achromatic transfer line) as a diagnostic line (see Fig. 1).

As the beam travels along the second part of the linac, the induced longitudinal wakefields reduce the energy chirp. Nonetheless, unless the full chirp is intentionally removed with a proper rf phasing, a residual correlation in the longitudinal phase space remains at the linac end and can be routinely detected at the DBD spectrometer. Approximately the same longitudinal correlation will remain and can be measured in the MBD line.

Figures (2a)-(2c) depict the measured longitudinal phase space of a typical electron bunch used during our experiment; one sees a significant, nearly linear energy chirp. The electron-beam longitudinal phase space is measured at FERMI by using a transverse rf deflector installed just before the bending of the DBD spectrometer [31]. An electron beam with similar characteristics has been used to perform a start-to-end simulation of the seeding process with the FEL code GINGER [32]. The simulated electron beam's longitudinal phase space at the undulator exit is shown in Fig. (2d). Both in the experiment and in the simulation, the external seed pulse was 170 fs (FWHM) long with $20 \mu \mathrm{J}$ energy at $260 \mathrm{~nm}$ wavelength to produce an FEL output pulse at $32.5 \mathrm{~nm}$. As a direct result of the seeding process and in part from the FEL gain in the radiator undulators, electrons that have interacted with the seed laser in the modulator undulator significantly change their energy distribution resulting in a local bump in the energy spread [Fig. (2d)]. Due to the original linear energy chirp, this modification of the electron-beam energy distribution, which is well localized in time and energy, manifests itself as a hole in the electron-beam energy spectrum [Fig. (2e)]. Despite the different energy of various parts of the electron beam interacting with the laser, the

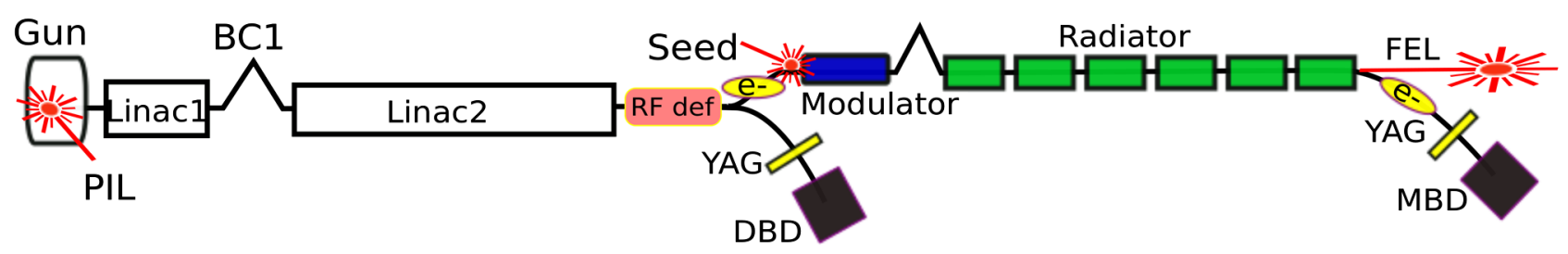

FIG. 1 (color online). FERMI layout includes the gun with the photoinjector laser (PIL), the linacs (linac 1 and 2), the bunch compressor (BC1), the undulators where the FEL is produced, and the spectrometer lines (DBD and MBD) for the beam energy characterization. A transverse rf deflector (RF def) installed before DBD allows one to measure the electron-beam longitudinal phase space in this location, the deflector is off during standard operations of the linac and during FEL. 

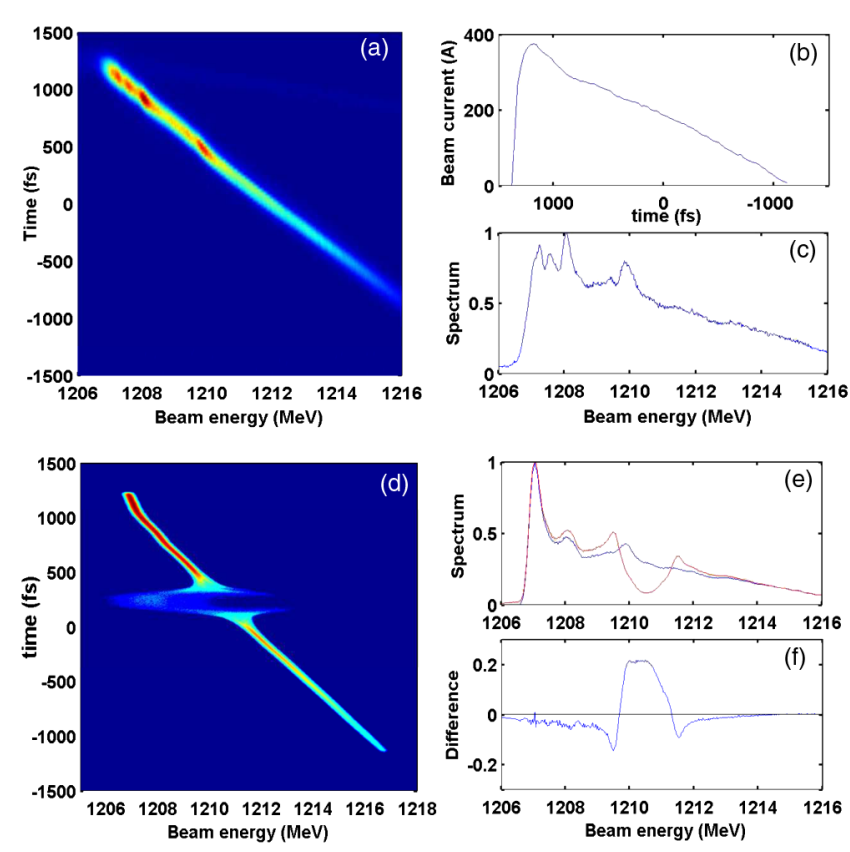

FIG. 2 (color online). Longitudinal phase space measured in DBD with the rf deflector (a), current profile (b), and energy spectrum (c) of the electron beam at the end of the LINAC before entering the undulators. Panels (d), (e), and (f) display numerical simulation results that have been done using the measured electron-beam properties. Panel (d) reports the phase space at the exit of the last radiator clearly showing the strong energy modulation in the spectrum. Panel (e) plots the electron-beam energy spectrum with (red) and without (blue) the seeding. Panel (f) reports the difference between the two.

energy transfer from the laser to the beam is guaranteed by the large bandwidth (a few percent) of the modulator undulator.

After passing through the undulators, the spent electron beam is sent to the main beam dump (MBD) line, as shown in Fig. 1. This line has a theoretical dispersion value of about $0.9 \mathrm{~m}$ which allows measurement of the energy spectrum by an intercepting yttrium-aluminum-garnet (YAG) screen. Examples of experimental MBD screen images and the corresponding derived electron-beam energy distributions are shown in Fig. 3. The spectra are similar to those measured at the linac end (DBD), as shown in Fig. (2a). A residual discrepancy between the two spectra is attributed to the longitudinal resistive wall wakefields in the undulator vacuum chamber [33].

Figure 3 shows MBD images and energy spectra for cases without [Figs. 3(a) and 3(b)] and with seeding [Figs. 3(c) and 3(d)] by an external laser. In the seeded case, a hole in the electron-beam energy distribution is clearly visible, as predicted by numerical simulations [see Figs. 2(d) and 2(f)]. Measurements of this hole can be used for characterization of several electron-beam properties.

As shown in Fig. 2(a), the electron beam has an almost linear energy chirp versus time. As a result, any temporal
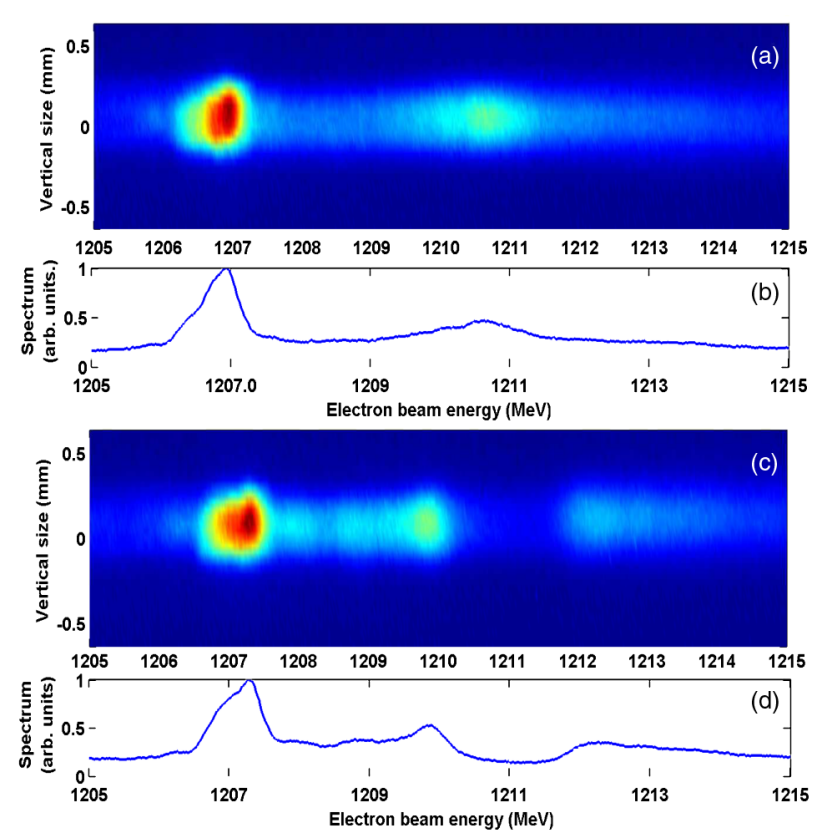

FIG. 3 (color online). Images of the electron beam [(a) and (c)] and corresponding spectrum [(b) and (d)] measured at MBD in the absence [(a) and (b)] and in the presence of the seed [(c) and (d)]. A hole in the electron-beam distribution is clearly evident at about $1.211 \mathrm{GeV}$.

shift of the seed with respect to the electron beam corresponds to a spatial shift of the hole in the energy spectrum. This property suggests one can obtain a very accurate measurement of the timing jitter between the seed and electron beam by recording the shot-to-shot location of the hole.

Accordingly, we acquired long sequences of spectra of the electron beam both with and without seeding [Fig. 4(a)]. Due to fluctuations in the accelerator, the
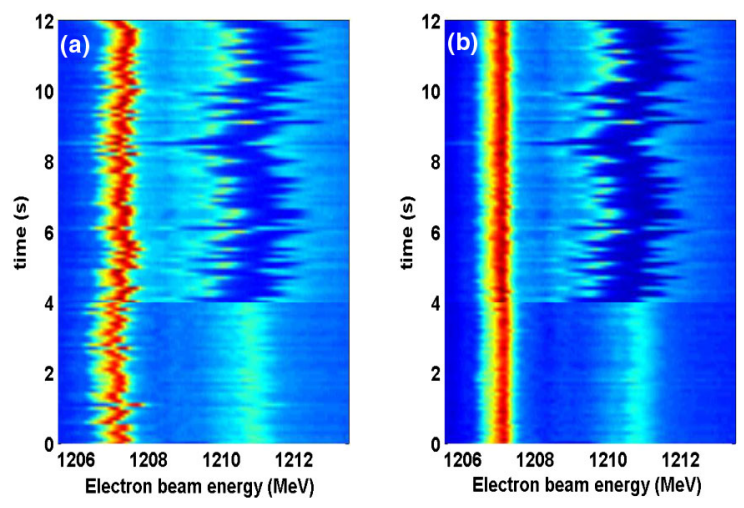

FIG. 4 (color online). (a) Sequence of the electron-beam spectra acquired in MBD. Spectra for time $<4$ s refer to electron beam that have not been interacting with the seed laser. Spectra for seeded beams $(t>4 \mathrm{~s})$ clearly show that in addition to the fluctuation in mean energy of the beam also the hole induced by the seed is fluctuating around $1211 \mathrm{MeV}$. Panel (b) shows the same data corrected for energy fluctuations. 
electron beam has not only arrival timing jitter but also shot-to-shot jitter in its final energy. Energy jitter changes the horizontal position of the spectrum on the MBD screen images as shown in Fig. 4(a) where both the mean and the peak energy fluctuate around their average values. For this particular experiment, the measured energy jitter was about $150 \mathrm{keV} \mathrm{rms}$. It is important to point out that, as long as the shape of the electron-beam energy spectrum does not change significantly from shot to shot, removal of the energy jitter does not affect the measurement of the time jitter. It should also be noted that the fluctuations of the position of the hole in the MBD spectra are significantly larger than those due to the energy jitter. Furthermore, there is no apparent correlation between the two.

In order to extract information on the hole dynamics from the data we removed the effect of energy fluctuation. The result of this data correction is shown in Fig. 4(b). Figure 5(a) shows the electron-beam spectrum, both in the absence (blue) and in the presence (red) of seeding. In Fig. 5(b) the difference between the two is shown. As a reference for the seed timing we considered the centroid of the positive pulse in Fig. 5(b), which corresponds to the seed laser-induced hole in the spectrum. It is important to note that, like in the case of the simulations, the charge removed from the interaction region (in energy) is moved towards higher and lower energies. A more detailed analysis shows an asymmetry in the charge energy redistribution around the seed-induced hole. A reproducible result of our experiment is that most of the charge removed by the seed is found in the lower energy side. This asymmetry, which is also present in the case of numerical simulations [Fig. 2(f)], is the result of the net extraction of electron-beam energy into FEL radiation. This is confirmed by additional numerical simulations (not reported here) showing a perfect symmetric energy redistribution of charge if the electron beam is analyzed downstream the modulator but before the radiator where FEL gain occurs.

A conversion of horizontal axis position into time is possible for the MBD images after a proper calibration. This has been done by measuring the average position of

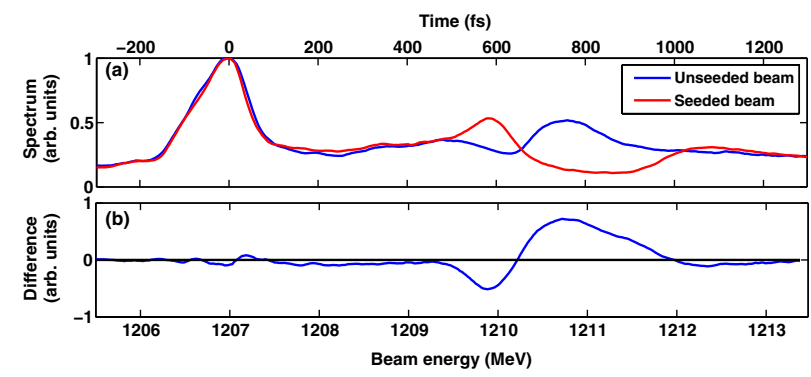

FIG. 5 (color online). (a) Profiles of the electron-beam density measured in MBD in case of a seeded (red) and unseeded (blue) beam. (b) Difference between the two profiles, showing the charge difference and the hole in the energy distribution produced by the seeding. the seed induced hole for various timings of the seed laser in a range \pm 100 fs around the working point. The resulting calibration of $0.22 \pm 0.02 \mathrm{pixel} / \mathrm{fs}$ has been used to define the horizontal axis of the top panel in Fig. 5. From this measurement one also obtains important information about the amount of electron-beam energy chirp. With the two calibrations, in $\mathrm{MeV}$ and fs, obtained for the CCD pixels one obtains a measure of the electron beam energy chirp of about $5 \mathrm{MeV} / \mathrm{ps}$ that is in agreement with measurement at the DBD with the rf deflector [Fig. 2(a)].

With this calibration factor and the correction for the mean energy fluctuation, we measured the relative timing between the electron beam and the seed laser for 200 individual shots. The timing evolution and its distribution are displayed in Fig. 6; removal of the mean timing leaves a relative jitter of less than $70 \mathrm{fs}$ rms. This result is in good agreement with predictions based on independent measurements of the separate systems. For the electron beam a time jitter of 40-50 fs has been measured [22]. For the seed laser, laboratory measurements suggest a jitter smaller than 30 fs [34] but no measurements have been made directly in the undulator region to date. It is important to point out that the reported scheme determines the relative timing jitter between the electron beam and the seed; this is the crucial information for a seeded FEL.

Additional information can be derived by this diagnostic method. For example, it produces a rough estimate of the seed pulse duration at the undulator. The length of the hole is related to the seed pulse duration, its strength, and the local value of the electron-beam chirp. In our experiment, since the electron-beam chirp is known together with the calibration obtained from previous analysis, we find the length for the laser induced hole to be about $180 \mathrm{fs}$ FWHM [Fig. 5(b)], which is in good agreement with the seed pulse duration measured in the laser room $(\simeq 170 \mathrm{fs}$ FWHM). This independent measurement of the seed laser duration is quite important because it removes all uncertainties about possible dispersion effects associated with the laser pulse passing through optical elements upstream of the modulator.

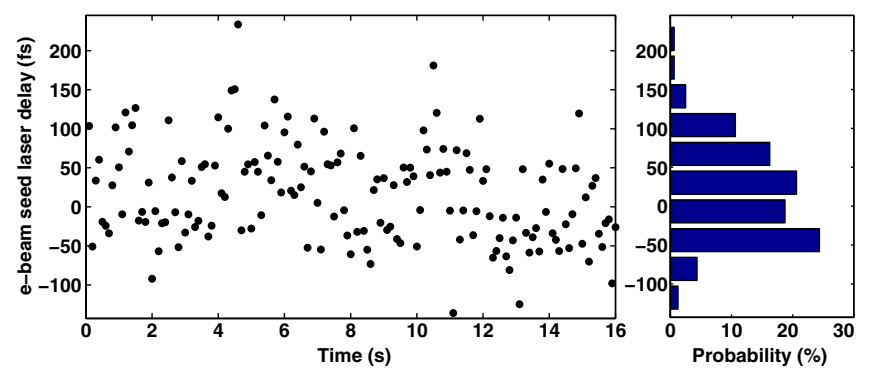

FIG. 6 (color online). (a) Evolution of the centroid of the hole produced by the seeding on a sequence of 200 consecutive electron bunches. (b) Distribution of the centroid positions characterized by an rms of $68 \mathrm{fs}$. 
An additional possible use for this diagnostic is a temporally resolved, quantitative characterization of the FEL process. Since the net transfer of energy from the electron beam to the FEL radiation pulse can be inferred from the electron energy distribution around the hole, in the case of a seeded FEL the scheme is suitable for giving an independent measure of the FEL flux as it was done at LCLS [5,35]. This technique opens the possibility to correlate the FEL gain with the temporally local e-beam energy distribution.

The proposed scheme could also be used for a more detailed characterization of the electron-beam properties. In particular, time-varying properties can be measured with this scheme without the need of a deflecting cavity. By scanning the seed laser on top of the electron beam and following the movement of the hole it is possible to measure the length of the electron bunch and the current profile. By keeping constant the seed properties (pulse length, intensity) and measuring the evolution of the hole length during the scan, one can determine details of the electron-beam energy chirp along the bunch. Finally, measurements of the hole behavior as a function of the seed laser power may allow an independent measurement of the local incoherent (i.e., slice) energy spread of the electron beam. Since measurements are based on the localized effects produced on the beam by an external laser, the scheme can produce very high temporal resolution by using short pulse lasers and extending the present capabilities based on rf-deflector methods. The use of the proposed method with few cycle lasers and a high resolution energy spectrometer could produce resolutions down to the few fs level.

An important requirement of the proposed scheme is the presence of a monotonic variation of the electronbeam energy with time at the diagnostic screen. Although a linear chirp makes analysis far simpler than more complicated (but still monotonic) chirps, linearity is not an absolute requirement. Moreover, in case the need for a monotonic variation is in conflict with other requirements driven by the optimization of the accelerator and/or the FEL, the proposed scheme could easily be implemented using a monoenergetic beam (e.g., to maximize FEL gain) upon which a linear energy chirp is added just before entering the beam dump [36]. Finally, our proposed method could be, in the case of seeded FELs, a cheaper alternative but also used in combination with deflecting cavities placed after the FEL undulators [37] that have made measurements of the localized FELinduced effects on the electron beam [38].

The authors thank the FERMI team for useful discussions and for their contribution in commissioning and operating the FEL. We acknowledge financial support from Italian Ministry of University and Research under Contracts No. FIRB-RBAP045JF2 and No. FIRBRBAP06AWK3.
[1] P. Ribic and G. Margaritondo, J. Phys. D 45, 213001 (2012).

[2] B. McNeil and N. Thompson, Nat. Photonics 4, 814 (2010).

[3] W. Ackermann et al., Nat. Photonics 1, 336 (2007).

[4] E. Allaria et al., Nat. Photonics 6, 699 (2012).

[5] P. Emma et al., Nat. Photonics 4, 641 (2010).

[6] T. Ishikawa et al., Nat. Photonics 6, 540 (2012).

[7] A. H. Lumpkin, Imaging Techniques for Transverse BeamProfile/Size Monitors, in Proceedings of the BIW2012, TUBP02, Newport, VA USA, 2012, edited by T. Satogata and J. Chew, http://accelconf.web.cern.ch/accelconf/ BIW2012/papers/tubp02.pdf.

[8] K. Wittenburg, Overview of Recent Halo Diagnosis and Non-Destructive Beam Profile Monitoring, in Proceedings of 39th ICFA Advanced Beam Dynamics Workshop on "High Intensity High Brightness Hadron Beams, Hb2006”, Tuaz01, Tsukuba, Japan, 2006, edited by Y. H. Chin and H. Yoshikawa, http://accelconf.web.cern.ch/ accelconf/abdwhb06/PAPERS/TUAZ01.pdf.

[9] A. H. Lumpkin, R. J. Dejus, and N. S. Sereno, Phys. Rev. ST Accel. Beams 12, 040704 (2009).

[10] A. Marinelli, M. Dunning, S. Weathersby, E. Hemsing, D. Xiang, G. Andonian, F. O'Shea, J. Miao, C. Hast, and J. B. Rosenzweig, Phys. Rev. Lett. 110, 094802 (2013).

[11] M. Castellano, V. A. Verzilov, L. Catani, A. Cianchi, G. Orlandi, and M. Geitz, Phys. Rev. E 63, 056501 (2001).

[12] M. Veronese, R. Appio, P. Craievich, and G. Penco, Phys. Rev. Lett. 110, 074802 (2013).

[13] R. De Monte, A. Borga, P. Craievich, M. Ferianis, G. Gaio, M. Predonzani, and M. Dal Forno, The FERMI@Elettra Cavity BPM System: Description and Commissioning Results, in Proceedings of DIPAC2011, MOOC03, Hamburg, Germany, 2011, edited by M. Marx, J. Chrin, I. Martin, R. Mueller, and V. RW Schaa, http://accelconf.web .cern.ch/accelconf/DIPAC2011/papers/mooc03.pdf.

[14] L. Froehlich, in DESY Report No. TESLA-FEL, 2005, Vol. 2005-02.

[15] S. Wesch, B. Schmidt, C. Behrens, H. Delsim-Hashemi, and P. Schmüser, Nucl. Instrum. Methods Phys. Res., Sect. A 665, 40 (2011).

[16] E. L. Saldin, E. A. Schneidmiller, and M. V. Yurkov, Nucl. Instrum. Methods Phys. Res., Sect. A 539, 499 (2005).

[17] D. Alesini, G. Di Pirro, L. Ficcadenti, A. Mostacci, L. Palumbo, J. Rosenzweig, and C. Vaccarezza, Nucl. Instrum. Methods Phys. Res., Sect. A 568, 488 (2006).

[18] G. Berden, S. P. Jamison, A. M. MacLeod, W. A. Gillespie, B. Redlich, and A. F. G. van der Meer, Phys. Rev. Lett. 93, 114802 (2004).

[19] B. Steffen et al., Phys. Rev. ST Accel. Beams 12, 032802 (2009).

[20] G. Angelova et al., Phys. Rev. ST Accel. Beams 11, 070702 (2008).

[21] M. Bionta et al., Opt. Express 19, 21855 (2011).

[22] P. Craievich, S. DiMitri, M. Milloch, G. Penco, and F. Rossi, Phys. Rev. ST Accel. Beams 16, 090401 (2013).

[23] G. Geloni, E. Saldin, E. Schneidmiller, and M. Yurkov, Opt. Commun. 281, 3762 (2008). 
[24] S. Schulz, M. K. Czwalinna, M. Felber, P. Predki, S. Schefer, H. Schlarb, and U. Wegner, Proc. SPIE Int. Soc. Opt. Eng. 8778, 87780R (2013).

[25] L. H. Yu, Phys. Rev. A 44, 5178 (1991).

[26] I. Ben-Zvi, K. Yang, and L. Yu, Nucl. Instrum. Methods Phys. Res., Sect. A 318, 726 (1992).

[27] B. Liu et al., Phys. Rev. ST Accel. Beams 16, 020704 (2013).

[28] E. Allaria et al., Nat. Photonics 7, 913 (2013).

[29] C. Bocchetta et al., FERMI@Elettra FEL Conceptual Design Report (Sincrotrone Trieste, Trieste, Italy, 2007), Vol. ST/F-TN-07/12.

[30] G. Penco et al., JINST 8, P05015 (2013).

[31] P. Craievich et al. (in preparation).

[32] W. M. Fawley, LBNL Technical Report No. LBNL-49625Rev.1, SLAC Report No. LCLS-TN-04-3, 2004.

[33] A. Lutman, R. Vescovo, and P. Craievich, Phys. Rev. ST Accel. Beams 11, 074401 (2008).
[34] P. Sigalotti, P. Cinquegrana, A. A. Demidovich, R. Ivanov, I. Nikolov, G. Kurdi, and M. B. Danailov, Proc. SPIE Int. Soc. Opt. Eng. 8778, 87780Q (2013).

[35] P. Emma, in Proceedings of the 23rd Particle Accelerator Conference, Vancouver, Canada, 2009 (IEEE, Piscataway, NJ, 2009), pp. 3115-3119.

[36] H. S. Kang, M. S. Chae, T. Ha, J. H. Hong, W. W. Lee, S. J. Park, and H. Yang, Control of Electron Beam Longitudinal Phase Space With a Novel Compact De-chirper for PAL-XFEL in Proceedings of the 35th International Free-Electron Laser Conference, MOICNO02, Manhattan, NY, USA, 2013, edited by C. Scholl and V. RW Schaa, http://accelconf.web.cern.ch/AccelConf/fel2013/talks/ moicno02_talk.pdf.

[37] Y. Ding, C. Behrens, P. Emma, J. Frisch, Z. Huang, H. Loos, P. Krejcik, and M.-H. Wang, Phys. Rev. ST Accel. Beams 14, 120701 (2011).

[38] C. Behrens et al. (in preparation). 